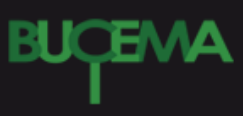

Bulletin du centre d'études médiévales d'Auxerre | BUCEMA

Hors-série $n^{\circ} 5$ | 2013

De Cluny à Auxerre, par la voie des "émotions". Un parcours d'historienne du Moyen Âge : Barbara H. Rosenwein

\title{
Les émotions et le moi chez quelques théologiens du haut Moyen Âge
}

Warren Pezé

\section{QpenEdition \\ Journals}

Édition électronique

URL : https://journals.openedition.org/cem/12538

DOI : $10.4000 /$ cem. 12538

ISSN : $1954-3093$

Éditeur

Centre d'études médiévales Saint-Germain d'Auxerre

Référence électronique

Warren Pezé, «Les émotions et le moi chez quelques théologiens du haut Moyen Âge », Bulletin du centre d'études médiévales d'Auxerre | BUCEMA [En ligne], Hors-série $n^{\circ} 5$ | 2013, mis en ligne le 08 janvier 2013, consulté le 02 mars 2023. URL : http://journals.openedition.org/cem/12538; DOI : https://doi.org/10.4000/cem.12538

Ce document a été généré automatiquement le 2 mars 2023

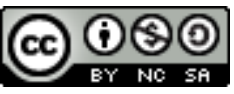

Creative Commons - Attribution - Pas d'Utilisation Commerciale - Partage dans les Mêmes Conditions 4.0 International - CC BY-NC-SA 4.0

https://creativecommons.org/licenses/by-nc-sa/4.0/ 


\title{
Les émotions et le moi chez quelques théologiens du haut Moyen Âge
}

\author{
Warren Pezé
}

1 Barbara Rosenwein a consacré en 2005 un article à la question suivante : «y avait-il un moi au haut Moyen Âge ? ${ }^{1}$ » Son but était de combler une lacune. Bien des auteurs s'étaient penchés sur la genèse de l'individualisme occidental. Certains pensaient que cet individualisme était né à la Renaissance, comme l'historien suisse Jacob Burckhardt ${ }^{2}$; d'autres estimaient qu'il avait des racines médiévales, comme Walther Ulmann et Colin Morris ${ }^{3}$. Malgré leurs divergences, les auteurs semblent s'accorder sur un point: on ne saurait parler d'individualisme avant le $\mathrm{xI}^{\mathrm{e}}$ siècle, avant le «beau " Moyen Âge. Le cas du haut Moyen Âge est le plus souvent réglé en début d'ouvrage ${ }^{4}$. B. Rosenwein résout donc deux problèmes. D'une part, elle comble une lacune en se consacrant exclusivement au haut Moyen Âge. D'autre part, elle propose une nouvelle méthodologie.

W. Ulmann avait adopté un point de vue juridique: il montrait que le statut de l'individu sortait renforcé de l'avènement des communes et de la féodalité fondée sur le contrat par rapport à ce qu'il appelait le "thème descendant» du haut Moyen Âge. C. Morris s'était surtout servi de sources littéraires: la littérature courtoise, l'autobiographie, la théologie, la satire, etc. B. Rosenwein, pour sa part, exploite des sources narratives. Sa thèse est la suivante : « au haut Moyen Âge, on était conscient de soi en tant qu'individu aux moments d'émotion intense. De tels instants se produisent souvent lors du passage d'un rôle à l'autre ${ }^{5}$.» Un de ses exemples, tiré du troisième livre des Dialogues de Grégoire le Grand, l'illustre particulièrement. Grégoire, un samedi saint, est victime de son estomac difficile et ne peut pas participer au jeûne de la communauté ; il se dit " affligé ", émotion causée par l'impossibilité de remplir son rôle dans sa communauté. À sa demande, son confrère Eleuthère, prie pour sa guérison, et l'obtient. Grégoire peut donc à nouveau jeûner, ce qui le met littéralement sens dessus dessous. "J'en suis ébahi : qu'est-ce que j'étais? Qu'est-ce que je suis maintenant ? ${ }^{6}$ " 
Comme conclut B. Rosenwein, «il n'est plus étranger à la communauté, mais fait à nouveau partie d'elle. Il décrit ce moment comme une prise de conscience de luimême ». Il s'agit donc d'une intense émotion réinterprétée d'après le changement de rôle : ce qui peut appeler deux commentaires.

3 D'abord, cette méthodologie concilie psychologie et sociologie grâce à la notion de rôle. La synthèse la plus récente et la plus efficace sur le rôle semble celle de Jean-Claude Kaufmann ${ }^{7}$. Reprenant notamment le livre fondateur du constructivisme de Peter Berger et Thomas Luckmann, La construction sociale de la réalité ${ }^{8}$, J.-C. Kaufmann affirme que le rôle social, dont le meilleur exemple reste le travail, sert de fixateur de la subjectivité. Par lui, le groupe confirme l'identité d'un individu. «L'identité subjective, mouvante et fragile, ne parvient à se fixer et à se densifier que par la prise de rôle, concrétisation et confirmation collective de soi ${ }^{9}$.» Dès lors, le meilleur indice d'individualisme et de réflexivité est sans doute la « distance au rôle ", qui repose sur la concurrence entre différents rôles et différents cercles de sociabilité. Autrement dit, la distance au rôle, quand on peut l'observer, permet d'évaluer le degré de holisme d'une société. Or, dans les cas décrits par l'article de B. Rosenwein, le changement de rôle produit un raptus émotionnel. Cela ne peut s'expliquer que par une certaine absence de réflexivité : d'absence de distance au rôle. Ce défaut de distance au rôle rejoint la notion holiste que l'on a traditionnellement du haut Moyen Âge, une période au cours de laquelle, schématiquement, la sociabilité serait davantage subie que choisie.

En second lieu, il semble qu'en ayant étudié ailleurs la communauté émotionnelle et en étudiant ici la représentation de soi, B. Rosenwein soit assez proche de la quadrature du cercle, c'est-à-dire de la production du sujet. Il est significatif que les exemples utilisés dans l'article sur " ego " au haut Moyen Âge soient ceux qui sont aussi utilisés dans Emotional communities: Grégoire le Grand, dont l'exemple est repris dans le chapitre Passions and power ${ }^{10}$. Dans le cas des communautés émotionnelles comme dans celui de l'individualisme, il est question de normes et de rôles, et les émotions servent à la subjectivité de miroir réfléchissant. Peut-être serait-il possible alors de faire la soudure entre ces notions que sont le sujet, la communauté et les émotions, d'une manière qui permettrait de cerner de manière unifiée la production du sujet par la société.

D'autres contributions à cette rencontre ont pour propos de développer la méthodologie de B. Rosenwein en matière d'émotions, ce qui me pousse à proposer autre chose. Je pensais d'abord tenter une enquête sur les émotions dans le style de B. Rosenwein, à la manière de son article de 2006 des Cahiers de Civilisation Médiévale, combinant " des études statistiques et des études lexicales des textes ${ }^{11}$ ", pour donner, par exemple, le spectre émotif d'une œuvre. Mais j'ai été attiré par une note de bas de page d'Emotional communities: « les communautés émotionnelles de saint Augustin et saint Jérôme sont mûres pour être explorées ${ }^{12}$. " L'intérêt de ce sujet est accru par un autre constat de B. Rosenwein : «il n'y a pas de théoricien des émotions au haut Moyen Âge ${ }^{13}$ ", mais seulement des théoriciens des vices et des vertus. De fait, les études sur les émotions au haut Moyen Âge s'appuient sinon sur des sources narratives, du moins sur des sources de nature pastorale : traités sur les vices et les vertus, règles monastiques, à la rigueur de l'exégèse, c'est-à-dire les sources dans lesquelles on parle le plus d'émotions, les Confessions d'Augustin ou les Conférences de Cassien par exemple. Il m'a semblé qu'un autre type de source mériterait qu'on y dépiste des sentiments : la théologie spéculative, en particulier celle qui porte sur les relations entre nature et 
grâce. Il faut rappeler d'abord que les émotions s'inscrivent à cette époque dans l'affrontement du bien et du mal.

\section{Dieu, le diable et les émotions}

\section{Bien, mal et émotions : le tournant patristique}

Comme l'expliquent Carla Casagrande et Silvana Vecchio, l'époque patristique voit se produire un changement de statut des émotions. Alors qu'on pouvait parler pour l'économie antique des passions d'une sagesse attentive, non au salut, mais au bien-être, on insiste désormais sur leur dimension salvifique ${ }^{14}$. "On reconnaît que les mouvements passionnels possèdent une valeur salvatrice ${ }^{15}$. » Dieu attend de l'homme un engagement affectif. Damien Boquet rappelle pour sa part qu'« il n'est pas de salut sans affectivité dans le christianisme. C'est par l'amour, le désir, la charité que le chrétien participe à son salut ${ }^{16}$ ». Les émotions sont donc interprétées en fonction des couples bien/mal, vertu/vice, salut/damnation, et surtout, Dieu/diable. Elles sont le résultat des relations entre trois personnes: l'homme, le diable et Dieu, et même davantage si l'on compte les anges. Quelle place parvient à occuper l'homme dans ce trio? N'est-il pas voué à se laisser toujours posséder par des êtres qui lui sont infiniment supérieurs? Et l'homme n'interprètera-t-il pas l'émotion, dont l'essence même est de s'imposer à la conscience, comme une marque, comme une preuve de cette possession? Comme l'écrit saint Ambroise au début du De fuga saeculi, «notre cœur et nos pensées ne sont pas en notre pouvoir; elles se répandent dans notre esprit et notre âme pour y jeter la confusion [...] qui est assez bienheureux pour toujours croître en son cœur? Mais qui peut y arriver sans l'aide de Dieu ? ${ }^{17}$ "

\section{Corps du Christ et corps du diable}

7 Quelques éléments peuvent servir d'introduction à ce problème: d'abord, le duel cosmique qui oppose pour les altimédiévaux le bien et le mal. On doit évoquer les actes du concile de Paris de 829 rédigés par Jonas d'Orléans. Jonas met en scène de manière très visuelle l'ecclésiologie altimédiévale, dans laquelle l'Église est certes le corps du Christ mais dans laquelle les réprouvés constituent aussi un « corps du diable ${ }^{18}$.

\begin{tabular}{|l|l|}
\hline Toute personne alors qui par des choses & Quisquis ergo per aliqua inlicita ex \\
interdites devient, de membre du Christ, & membro Christi se fecit membrum \\
membre du diable, qu'elle sache qu'elle & diaboli noverit se non in corpore Christi, \\
n'est plus dans le corps du Christ mais & sed in corpore esse diaboli. Proinde \\
dans celui du diable. Il devient alors & necesse est, ut corpori Christ, a quo astu \\
nécessaire qu'elle ne néglige pas de se & diabolico separatus est, se incunctanter, \\
rendre le plus vite possible au corps du & dum tempus penitentiae in promptu \\
Christ dont elle a été séparée par la & habetur, restituere non neglegat. \\
tentation diabolique, tant qu'elle a à & \\
disposition le temps de la pénitence. &
\end{tabular}

8 Dans cette représentation du monde, l'homme fait, en toute circonstance, partie d'un grand corps, invisible mais efficace. Il n'existe pas d'entre-deux, d'espace moralement neutre. Ce qui sépare du corps du Christ, dit Jonas, c'est la «tentation diabolique » : non 
seulement l'homme est impliqué de force dans un conflit cosmique, mais en plus les deux protagonistes se le disputent.

\section{Les émotions sur l'échelle de la tentation}

9 Cette bataille n'a rien d'extérieur et a pour champ ce que l'homme a de plus intime : ses pensées, ses émotions. Il n'existe pas de for intérieur protégé du bien et du mal. C'est là une tendance qui a été mise en lumière par D. Boquet ${ }^{19}$. En se penchant sur les trois degrés de la tentation, à savoir suggestion, délectation, consentement, il montre que le mal tend chez certains auteurs à débuter dès la suggestion. Le fait même d'être tenté, de concevoir une certaine pensée ou un certain sentiment, est perçu comme un péché. On peut citer par exemple la Regula pastoralis de Grégoire le Grand ${ }^{20}$ :

« Nous avons appris par l'exemple de notre premier père que toujours le mal de la faute est perpétré selon trois degrés: la suggestion, la délectation, le consentement; le premier par l'ennemi, le second par la chair, le troisième par l'esprit. »

10 Toujours, dit Grégoire, la suggestion vient du diable; toujours, elle sera considérée comme une faute. C'est là un point de vue assez extrême, comme l'explique D. Boquet, mais il fit des émules. Dans un tel cadre mental, il devient nécessaire de disséquer ses moindres pensées et ses moindres sentiments pour y séparer au scalpel le bien du mal, pour distinguer la présence du Sauveur de celle de l'Ennemi. C'est ce que concluait M. Foucault de son étude sur la chasteté chez Cassien : « il s'agit de débusquer en soi la puissance de l'Autre, de l'Ennemi, qui s'y cache sous les apparences de soi-même : parce qu'il s'agit de mener contre cet Autre un combat incessant dont on ne saurait être vainqueur sans le secours de la Toute-Puissance ${ }^{21}$.» Il me semble que ce diagnostic met bien en valeur la façon dont, si je puis dire, l'ordre de l'affect est désubjectivé au profit de puissances supérieures et invisibles qui le manipulent.

\section{Remplacer les anges déchus}

11 Ainsi, les émotions seraient la partie émergée d'une possession : en débusquant en soi «le secours de la Toute-Puissance » dont parle Foucault, l'individu discerne, et donc stimule, des pensées et des émotions moralement positives, vertueuses, dont il ne se pense pas la cause. Bien plus : il tend à devenir autre, à se considérer comme une chose agie. Cela rejoint la croyance, propre au haut Moyen Âge, que les hommes ont été créés pour remplacer les anges déchus ${ }^{22}$. Beaucoup, Augustin, dans l'Enchiridion, Grégoire, dans les Homélies sur l'Evangile, Isidore dans les Sententiae, Hincmar dans l'Ad simplices, soutiennent que l'homme a été créé pour remplacer le numerus clausus des anges déchus. Je cite en exemple l'Enchiridion d'Augustin ${ }^{23}$ : 


\begin{tabular}{ll|l|}
\hline Dieu, créateur et organisateur de & $\begin{array}{l}\text { Placuit itaque universitatis Creatori } \\
\text { l'univers, décida que puisque toute la }\end{array}$ & atque moderatori Deo ut quoniam non \\
foule des anges n'avait pas péri en & tota multitudo angelorum Deum \\
l'abandonnant, celle qui avait péri & deserendo perierat, ea quae perierat in \\
demeurerait dans sa perdition & perpetua perditione remaneret; quae \\
perpétuelle et que celle qui était restée & autem cum Deo illa deserente perstiterat \\
auprès de lui jouirait de la certitude & de sua certissime cognita semper futura \\
absolue de sa joie future, mais qu'en & felicitate gauderet; alia vero creatura \\
revanche, une autre créature rationnelle, & rationalis, quae in hominibus erat, \\
qui consistait dans les hommes et qui & quoniam peccatis atque supplicis et \\
avait péri toute entière dans le péché & originalibus et propriis tota perierat, ex \\
originel et le péché propre, suppléerait & eius parte reparata quod angelicae \\
en nombre ce que la chute du diable & societati ruina illa diabolica minuerat \\
avait retranché à la société diabolique. & suppleretur. \\
\hline
\end{tabular}

Les élus sont donc destinés à remplacer le nombre des anges déchus. Il reste à citer une phrase plutôt lucide de C. Morris au sujet du haut Moyen Âge : « le but de l'homme n'est pas humain mais angélique : il n'est pas de se réaliser, mais de devenir quelque chose de différent ${ }^{24}$.» Dans cet élan de purification, il est certain que pensées et émotions, processus en partie involontaires que la philosophie classique distingue mais que l'on sait au moins depuis Antonio Damasio indissociables, jouent un rôle essentiel ${ }^{25}$.

\section{« La chambre du Christ, c'est un cœur droit et bon »}

\section{L'augustinisme : nature et grâce}

13 En somme, les émotions tiennent dans la cosmologie chrétienne du haut Moyen Âge une place de choix. Nous avons vu que ces intrusions de puissances surnaturelles contribuaient à l'incorporation de l'individu dans une altérité qui le dépasse (corps du Christ, corps du diable), et tendaient même à transformer sa propre nature (avec la substitution du nombre angélique). Il faut maintenant situer ces émotions dans l'économie plus générale de la grâce. Celle-ci correspond peu ou prou à la doctrine augustinienne qui triomphe du pélagianisme au concile d'Orange en 529. Dans l'augustinisme, la nature humaine est irrévocablement corrompue par le péché originel de telle sorte que seul Dieu puisse la restaurer et la rendre de nouveau capable du bien. Comme le résumait Étienne Gilson, «le Dieu d'Augustin guérit une nature ${ }^{26}$ ». Il n'est aucun bien qui ne soit causé par Dieu: dès lors, toute émotion bonne est agie en l'homme par Dieu.

\section{La Psychomachie de Prudence}

Pour illustrer cette conception instrumentale de la nature humaine, j'aimerais citer des textes qui dépeignent le cœur comme un lieu que Dieu doit habiter. Le premier de ces textes est très connu : il s'agit de la Psychomachie de Prudence, poète chrétien mort à Rome vers 410 , et dont Gennade a donné la première biographie dans le De viris illustribus. La Psychomachie décrit le combat allégorique des vices et des vertus. À la fin du combat, la Concorde et la Foi exhortent les vertus à construire le Temple de Dieu, comme Salomon l'avait fait après les guerres davidiques. Mais, sous le régime néotestamentaire qu'illustre l'épisode de la Samaritaine en Jean 4, le Temple, c'est 
dorénavant chaque individu. On voit donc Concorde et Foi édifier, des vers 823 à 887 , le temple de l'âme. Après que les bâtisseurs ont inscrit sur les portes les noms des douze apôtres (référence à Apocalypse 21) voici ce que décrit Prudence ${ }^{27}$ :

\begin{tabular}{|c|c|}
\hline $\begin{array}{l}\text { nscriptions, l'Esprit sollicite les } \\
\text { ecrets de l'âme,/ appelle dans le } \\
\text { s sentiments choisis ;/ et du côté } \\
\text { nouit la nature humaine, } \\
\text { le dans tout le corps/ une } \\
\text { le force, l'Esprit pénètre par } \\
\text { tes jusqu'à l'autel/ du cœur, }\end{array}$ & $\begin{array}{l}\text { Spiritus his titulis arcana recondita } \\
\text { mentis } \\
\text { Ambit, et electos uocat in praecordia } \\
\text { sensus ; } \\
\text { Quaque hominis natura uiget, quam } \\
\text { corpore toto } \\
\text { Quadrua uis animat, trinis ingressibus } \\
\text { aram } \\
\text { Cordis adit, castisque colit sacraria uot }\end{array}$ \\
\hline
\end{tabular}

15 Cette topographie est instructive. Les quatre côtés du temple représentent en général l'ordre naturel et ici, les quatre âges de la vie. Les portes, qui sont trois par côté, représentent évidemment Dieu. C'est donc par les portes divines que l'Esprit pénètre jusqu'au cœur qui est l'autel, le Saint des Saints, où a lieu le sacrifice. Et ce sacrifice, ce sont les «sentiments choisis». On se rappelle le Psaume 50 : sacrificium dei spiritus contribulatus, cor contritum. Autrement dit, le cœur est un lieu que Dieu habite et où il suscite lui-même des sentiments vertueux.

\section{L'anonyme De camara Christi}

16 Le second exemple est un texte à la fois court et obscur, le De camara Christi. Ce petit document édité par Bernhard Bischoff date d'avant le viII siècle; il s'agit d'une description allégorique du cœur comme chambre $d u$ Christ ${ }^{28}$. Il a été transmis par plusieurs manuscrits où il occupe à peine un folio.

\begin{tabular}{|l|l|}
\hline La chambre du Christ c'est un cœur droit & De Camara Christi cum hominem dei hic \\
et bon; ses fondations, c'est la foi ; sa & est cor rectum et bonum \\
hauteur, l'espérance; sa largeur, la & Fundamentum eius est fides. \\
charité [...] son fauteuil, c'est la sérénité & Altitudo eius spes. \\
d'esprit; son épouse, c'est une âme & Latitudo eius caritas. [...] \\
sainte; ses chambrières sont les vertus & Cathedra Christi est serenitas mentis. \\
spirituelles; la première est la sainte & Sponsa Christi est sancta anima. \\
charité, qui dirige la chambre du Christ; ; & Camarariae Christi virtutes spiritales \\
la deuxième est la sainte humilité, qui & sunt. \\
garde le trésor dans la chambre [...] la & Prima sancta caritas dicta est, illa regit \\
quinzième est la persévérance, qui & camaram Christi. \\
nourrit toutes les précédentes jusqu'à la & Secunda est sancta humilitas, illa est \\
perfection. Que Dieu fasse qu'il trouve & thensauraria in camara. [...] \\
en nous une telle maison! & Quinta décima est sancta perseverantia, \\
& illa nutrix earum est usque ad finem \\
perfectum. & Prestet nobis Dominus, ut inveniat in \\
& nobis talem mansionem, qui vivit et \\
& régnât in secula seculorum. Amen. \\
\hline
\end{tabular}

Chaque objet domestique est réinterprété comme une propriété spirituelle. Ce petit extrait est finalement de même substance que la Psychomachie (B. Bischoff plaide en 
faveur d'une inspiration directe) : le cœur humain est un lieu où se trouvent toutes sortes d'instruments (fauteuil, table, etc.), symboles des vertus que Dieu a à disposition.

Il me semble probable que l'origine de cette conception instrumentale du cœur soit une expérience sensible assez commune : le fait de ne pas contrôler ses émotions. À la fin de la Psychomachie (vers 908-9), Prudence adresse une prière au Christ dans laquelle on lit quelque chose de révélateur sur cette passivité :

\begin{tabular}{|l|l|}
\hline Avec leurs divers esprits, la lumière et les & $\begin{array}{l}\text { Spiritibus pugnant variis lux atque } \\
\text { tenebrères combattent }\end{array}$ \\
$\begin{array}{l}\text { et notre substance double nourrit des } \\
\text { forces contraires. }\end{array}$ & $\begin{array}{l}\text { Distantesque animat duplex substantia } \\
\text { vires. }\end{array}$ \\
\hline
\end{tabular}

Ainsi l'arbitraire des sentiments peut-il stimuler l'idée d'un arbitraire de la grâce.

\section{Les émotions et le débat sur la grâce}

\section{Indurabo cor Pharaonis (Exode 4, $21 ; 7,3 ; 14,4$ )}

Dans la controverse entre augustiniens et pélagiens, le point crucial est le suivant : la nature humaine est-elle encore, après la chute, capable de faire le bien ? Augustin répond que sans la grâce, non. En apparence, cette controverse est bien abstraite. Ce n'est pas le cas. J'aimerais me servir, pour en faire ressortir les émotions, d'un passage de la Bible très largement commenté : l'endurcissement du cœur de Pharaon. Entre Exode 4 et 14, Moïse et Aaron s'efforcent de faire sortir d'Égypte le peuple hébreu. Avant de les laisser partir, Pharaon supporte que s'abattent dix plaies sur le pays. Après cela, pris de remord, il se lance à leur poursuite et engloutit son armée dans la mer Rouge. Apparemment, ce n'est pas totalement de sa faute : à trois reprises, en Exode 4, $21,7,3$ et 14,4 , l'Éternel dit à Moïse "j'endurcirai le cœur de Pharaon", endurcissement indispensable à ce que l'Égypte soit punie et subisse intégralement les dix plaies. Cet épisode a posé problème. Dieu fausse la responsabilité de Pharaon en faisant de lui le persécuteur. Cet endurcissement a une connotation émotionnelle transparente: les commentateurs ont tous compris «l'endurcissement» comme l'incapacité d'aimer.

\section{Les émotions dans l'anonyme pélagien De induratione cordis pharaonis}

21 Un pélagien anonyme, que l'on a cru un temps être Pélage lui-même, a consacré à la question un traité De induratione cordis pharaonis, sans doute écrit vers 404-405 ${ }^{29}$. L'auteur propose une interprétation pélagienne de plusieurs passages litigieux de la Bible, et notamment, l'endurcissement de Pharaon. Le fond de l'argument est que l'endurcissement est un châtiment a posteriori. L'Éternel a endurci pour le châtier un cœur déjà coupable: ce sera pour nous le prétexte à une petite recherche sur les émotions. Les pélagiens sont intuitivement ceux qui accordent le plus à la nature et le moins à la grâce : quelle est leur idée de l'action de Dieu et du diable sur les émotions ? Dans le paragraphe vingt-quatre, l'auteur étudie qui est responsable du péché de Pharaon avant l'endurcissement. Il explique alors que le cœur est habité ou bien par Dieu, ou bien par le diable, qui y règnent en maîtres. 
Il y a trois suspects: Dieu, la volonté personnelle et le diable, si la volonté lui consent. [...] mais si c'est le diable, I'homme n'a pas pu en rendre responsable sa propre folie; car sa volonté a donné la main et a permis d'habiter dans son cœur au diable qui endurcit tout cœur qu'il pénètre selon sa propre volonté.
Tres sunt a quibus suspicio est: Deus, propria voluntas et diabolus, si tamen diabolo voluntas consenserit [...] si vero a diabolo, ipse nihilominus suae imputavit dementiae, cuius voluntas manum diabolo porrexit et sui cordis habitatorem permisit effici, qui ad suam voluntatem indurat in quod ingressus fuerit cor.

Pour le pélagien anonyme, même s'il existe toujours la barrière du consentement qui cède à qui il veut, le cœur n'est un réduit inaccessible ni pour Dieu, ni pour le diable. La seule liberté qui reste, c'est de choisir qui va habiter son cœur pour y susciter les bonnes émotions. Dans le paragraphe vingt-huit, l'auteur décrit comment Dieu et le diable se succèdent dans le cœur de l'individu.

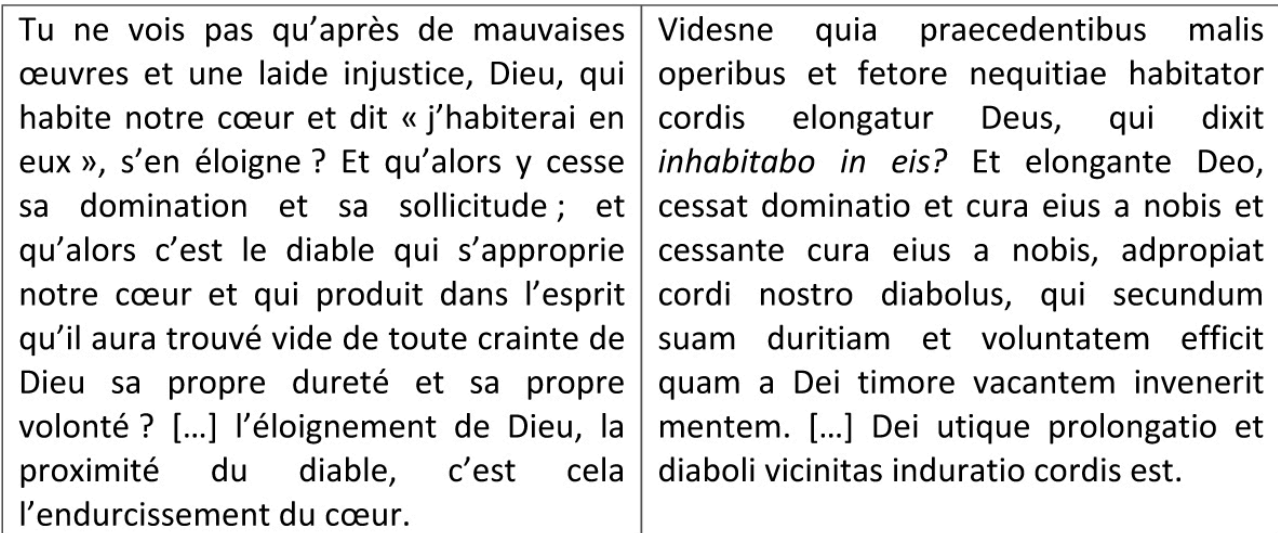

Dieu et le Diable alternent dans le cœur de l'homme. Ils transforment alors la nature de leur hôte et suscitent en lui bonnes ou mauvaises émotions. C'est ce que nous apprend une très belle métaphore plus loin. Pour l'auteur, Dieu transforme le cœur en or alors que le diable le transforme en boue. Mais, certaines circonstances, que l'auteur appelle tribulatio, le tourment, soumettent le cœur au feu, à la chaleur intérieure. B. Rosenwein a montré, dans Emotional communities, que pour Grégoire le Grand, le désir était associé à la chaleur ${ }^{30}$. Au contact de ce feu, les coeurs de boue possédés par le Diable cuisent et s'endurcissent alors que les cœurs d'or possédés par Dieu s'amollissent, explique le paragraphe trente-deux. 
Dieu avait prévu que le cœur de Pharaon serait approché du feu du tourment et, comme de la boue, s'endurcirait [...] II changea l'or en boue, et le Seigneur horrifié la lança comme une masse de boue dans le feu, autrement dit il permit qu'il passe de sa protection au pouvoir du diable. [...] mais le personnage du juste, qu'on compare à l'or, même s'il tire son origine de la terre, comme l'impie, est transformé en or en croyant au Dieu du ciel et en mettant de tout son cœur son espérance en lui ; il sera gardé parmi les trésors des cieux; à l'instar du bronze, quand on l'approche du feu du tourment, non seulement il ne durcit pas mais il s'amollit et ressent la main de l'artisan qui le travaille ; il reconnaît qu'il tire profit du marteau qui le frappe pour le corriger et non pour le briser.
Praevidebat ergo Deus cor Pharaonis in liberatione Israel ad ignem tribulationis admoveri et admotum utpote lutum indurari ab igne. [...] aurum in lutum mutavit, quod Dominus perhorrescens quasi massam luti de manu in ignem iactavit, id est, de sua protectione in diaboli potestatem verti permisit. [...] Justi vero persona qui auro adsimilatur, licet de materia terrae sumat originem sicut impius, Deum tamen caeli credendo et in eo spem suam ex toto corde ponendo, aurum efficitur, in caelorum thesauris conservandus ; ut aes, cum admotum fuerit ad ignem tribulationis, non solum minime induratur sed etiam mollescit et sentit artificis manum et se multum proficere et percutientis se malleo corrigi potius quam frangi agnoscit.

Dans les moments de détresse intime, l'élu s'adoucit et le réprouvé s'endurcit, comme or et boue au contact du feu; et c'est là entièrement l'œuvre des forces surnaturelles qui l'habitent.

On retient de ce traité qu'en dépit de son attachement pélagien, il réduit au strict minimum la responsabilité humaine envers les émotions. Tout ce qui vient du cœur vient ou du diable ou de Dieu: il appartient seulement à l'homme de choisir qui l'habite. En vertu d'une sorte d'acte libre originel totalement théorique, l'homme se retrouve habité ou par Dieu ou par le Diable qui orientent ses pensées et ses sentiments de telle sorte qu'il semble impossible de s'en débarrasser.

\section{Les notes de lecture de Ratramne et Florus : la « communauté émotionnelle » d'Augustin}

Nous venons d'avoir un aperçu pélagien de la question des émotions. Pour leur part, les réflexions d'Augustin sur le fait que tout mouvement vertueux de l'âme est causé par Dieu sont nombreuses et éparpillées. Je propose, dans un dernier temps, de profiter de ce thème pour parler de communauté émotionnelle. Dans Emotional communities, B. Rosenwein parle de ces communautés émotionnelles comme de «communautés sociales ", mais aussi, de communautés "textuelles »: «mais il peut aussi s'agir d'une communauté textuelle, créée et consolidée par des idéologies, des doctrines, des présupposés communs ${ }^{31}$. " J'aimerais suggérer l'existence d'une telle communauté, celle des clercs de tendance augustinienne, en utilisant les notes de lecture prises par deux célèbres clercs du $\mathrm{IX}^{\mathrm{e}}$ siècle, Ratramne, écolâtre de Corbie mort vers 870 , et Florus Diacre, écolâtre du chapitre de Lyon mort en 860 . Ces notes prises sur des textes d'Augustin sont un témoin précieux de l'assimilation d'idées augustiniennes par les Carolingiens.

27 Voici par exemple les notes prises par Ratramne dans les manuscrits latins 12205 et 12210 de la Bibliothèque nationale de France $(\mathrm{BnF})$ pour préparer son $D e$ 
praedestinatione en 849 . Ratramne accumule les notes sur le fait que c'est de Dieu que viennent les bonnes pensées et les différentes vertus.

\begin{tabular}{|c|c|}
\hline $\begin{array}{l}\text { BnF latin } 12205, \text { f. } 13 r \\
\text { que la bonne pensée est une grâce de } \\
\text { Dieu }\end{array}$ & $\begin{array}{l}\text { BnF latin } 12205, \text { f. } 13 \mathrm{r} \\
\text { quod cogitatio bona gratia dei sit }\end{array}$ \\
\hline $\begin{array}{l}\text { BnF latin } 12205, \text { f. } 21 \mathrm{r} \\
\text { Que la charité ne procède que de Dieu }\end{array}$ & $\begin{array}{l}\text { BnF latin } 12205, \text { f. } 21 \mathrm{r} \\
\text { Quod caritas nonnisi ex deo sit }\end{array}$ \\
\hline $\begin{array}{l}\text { BnF latin } 12210, f .73 r \\
\text { La foi est un don de Dieu } \\
\text { Que le commencement de la foi est un } \\
\text { don de Dieu }\end{array}$ & $\begin{array}{l}\text { BnF latin } 12210, \text { f. } 73 r \\
\text { Fides donum dei est } \\
\text { Quod initium fidei donum dei sit }\end{array}$ \\
\hline $\begin{array}{l}\text { BnF latin } 12210, f .74 r \\
\text { Que la bonne pensée procède de Dieu }\end{array}$ & $\begin{array}{l}\text { BnF latin } 12210, \text { f. } 74 \mathrm{r} \\
\text { Quod cogitatio bona ex deo est }\end{array}$ \\
\hline $\begin{array}{l}\text { BnF latin } 12210, f .80 v \\
\text { Que la grâce divine n'est repoussée par } \\
\text { la dureté d'aucun cœur }\end{array}$ & $\begin{array}{l}\text { BnFlatin } 12210, \text { f. } 80 \mathrm{v} \\
\text { Quod divina gratia nullo duro corde } \\
\text { respuitur }\end{array}$ \\
\hline
\end{tabular}

On dira que la cogitatio, ce ne sont pas les émotions; mais on pourrait répondre, bien malin qui les distinguera! On pourrait aussi citer les Confessions d'Augustin (IX, 3) pour montrer que pensée et émotion sont difficilement discernables : «Tu avais transpercé notre cœur de flèches par ta charité, nous portions tes paroles fichées dans les entrailles, et les exemples de tes esclaves, accumulés dans les replis de nos pensées, nous brûlaient. » Mais les émotions sont encore plus prégnantes dans le cas de Florus, écolâtre de Lyon extrêmement soucieux d'éthique. Pour préparer ses traités prédestinatiens, Florus a amplement utilisé le Ms. Lyon, Bibliothèque municipale, 608, copié sous Leydrade et contenant quasiment tous les opuscules d'Augustin sur la prédestination.

\begin{tabular}{|c|c|}
\hline $\begin{array}{l}\text { Lyon, Bibliothèque municipale, } 608, \\
\text { f. } 133 r \\
\text { Dieu fait ce qu'il veut dans les cœurs des } \\
\text { hommes, ou bien par miséricorde, ou } \\
\text { bien par jugement, en inclinant leur } \\
\text { volonté s'il le faut à se comporter } \\
\text { humainement ou divinement; cela a été } \\
\text { inutilement reproché au saint docteur }\end{array}$ & $\begin{array}{l}\text { Lyon, Bibliothèque municipale, } 608 \text {, } \\
\text { f. } 133 \mathrm{r} \\
\text { agere deum in cordibus hominum quod } \\
\text { vult vel misericordia vel iudicio inclinando } \\
\text { eorum si oportet voluntatem sive ad } \\
\text { humana sive ad divina peragenda, quod } \\
\text { in verbis sancti doctoris inepte est } \\
\text { repraehensum }\end{array}$ \\
\hline $\begin{array}{l}\text { Lyon, Bibliothèque municipale, } 608 \text {, } \\
\text { f. } 134 \mathrm{r} \\
\text { que le commencement, le progrès et la } \\
\text { perfection de la foi proviennent de Dieu, } \\
\text { comme pour les autres vertus: } \\
\text { continence, patience, justice, etc. }\end{array}$ & $\begin{array}{l}\text { Lyon, Bibliothèque municipale, } 608 \text {, } \\
\text { f. } 134 \mathrm{r} \\
\text { Quod initium et incrementum et } \\
\text { perfectionem fidei ex deo esse, sicut et } \\
\text { aliarum virtutum continentiae, } \\
\text { patientiae, iustitiae et caetera }\end{array}$ \\
\hline $\begin{array}{l}\text { Lyon, Bibliothèque municipale, } 608 \text {, } \\
\text { f. } 149 \mathrm{v} \\
\text { que l'appel éternel de Dieu, par lequel il } \\
\text { appel ses élus, se produit non seulement } \\
\text { par une parole extérieure mais par son } \\
\text { inspiration intérieure et immuable }\end{array}$ & $\begin{array}{l}\text { Lyon, Bibliothèque municipale, } 608 \text {, } \\
\text { f. } 149 \mathrm{v} \\
\text { Quod vocatio dei aeterna qua electos } \\
\text { suos vocat non sermone tantum } \\
\text { extrinsecus sed intima et immutabili eius } \\
\text { inspiratione fiat }\end{array}$ \\
\hline $\begin{array}{l}\text { Lyon, Bibliothèque municipale, } 608 \text {, } \\
\text { f. } 166 \mathrm{r} \\
\text { le sentiment propre au fait de prier, } \\
\text { c'est-à-dire demander, quémander et } \\
\text { frapper à la porte, est un don de l'esprit } \\
\text { saint qui intercède pour nous par des } \\
\text { gémissements inexprimables, en faisant } \\
\text { gémir et demander les fidèles }\end{array}$ & $\begin{array}{l}\text { Lyon, Bibliothèque municipale, } 608 \text {, } \\
\text { f. } 166 \mathrm{r} \\
\text { ipsum orandi id est petendi, quaerendi et } \\
\text { pulsandi affectum, donum esse spiritus } \\
\text { sancti qui postulat pro nobis gemitibus } \\
\text { inenarrabilibus cum fideles postulantes } \\
\text { ac gementes facit }\end{array}$ \\
\hline
\end{tabular}


Nous voyons comment, en se transmettant de texte en texte, la pensée d'Augustin a formé une communauté émotionnelle chevauchant les générations. Ces clercs de diverses époques avaient appris à attribuer à Dieu toutes les émotions bonnes qui les animaient : la notion que ces sentiments revenaient en définitive à Dieu tisse entre eux les liens d'une communauté émotionnelle invisible. Nous en revenons donc, après un détour par l'individualisme, à ces communautés que nous a appris à discerner B. Rosenwein et qui sont l'objet des communications suivantes.

\section{NOTES}

1. B. RoSENWEIN, «Y avait-il un moi au haut Moyen Âge ? ", Revue Historique, 631, 2005/1, p. 31-52.

2. J. BURCKHARDT, Die Kultur der Renaissance in Italien, Basel, 1860.

3. W. ULMANN, The individual and society in the middle ages, London, 1967 et C. MORRIS, The discovery of the individual, London, 1972.

4. Comme dans C. MORRIS, The discovery of the individual, op. cit., p. 30-31.

5. B. ROSENWEIN, «Y avait-il un moi », op. cit., p. 43.

6. Ibid., p. 46.

7. J.-C. KAUfMANN, Ego, pour une sociologie de l'individu, Paris, 2004.

8. P. BERGER et T. LUCKMANN, La construction sociale de la réalité, Paris, 1986.

9. Ibid., p. 194.

10. B. ROSENWEIN, Emotional communities in the early Middle Ages, Ithaca, 2006, p. 79-100.

11. B. ROSENWEIN, « Histoire de l'émotion : méthodes et approches ", in La médiévistique au XXe siècle. Bilan et perspectives, Poitiers, 2006 (Cahiers de civilisation médiévale, 49) p. 33-48 (p. 45).

12. Op. cit., p. 2, n. 5 .

13. B. ROSENWEIN, « Emotion words », in P. NAGY et D. BOQUET (dir.), Le sujet des émotions au Moyen Âge, Paris, 2008, p. 93-106 (p. 97).

14. C. CASAGRANDE et S. VECCHIO, «Les théories des passions dans la culture médiévale », ibid., p. 107-22.

15. Ibid., p. 110.

16. D. BOQUET, « des racines de l'émotion, les préaffects et le tournant anthropologique du XII siècle ", ibid., p. 163-86 (p. 181).

17. AMBRoISE DE MILAN, De fuga saeculi, $\S 1$ et 2, Patrologia latina 14, col. 569 : « Non enim in potestate nostra est cor nostrum et nostrae cogitationes quae improviso effusae mentem animumque confundunt, atque alio trahunt quam tu proposueris, ad saecularia revoquant, mundana inserunt, voluptuaria ingerunt, illecebrosa intexunt ; ipsoque in tempore quo elevare mentem paramus, insertis inanibus cogitationibus, ad terrena plerumque dejicimur. Quis autem tam beatus qui in corde suo semper ascendat, sed hoc sine auxilio divino qui fieri potest?»

18. Concile de Paris (829); MGH Concilia II, 2, éd. A. WERMINGHOFF, Hanover/Leipzig, 1906, p. 610.

19. D. BOQUET, « Des racines de l'émotion ", op. cit.

20. GRÉGOIRE LE GRAND, Regula pastoralis, éd. et trad. B. JUDIC, Paris, 1992, p. 475.

21. M. Foucault, « Le combat de la chasteté », Communications 35, 1982, p. 15-25 (p. 23).

22. Cf. y. CONGAR, L'ecclésiologie du haut Moyen Âge, Paris, 1968, p. 103-105. 
23. AUGUSTIN D'HIPPONE, Enchiridion de fide, spe et caritate ad Laurentium, $§ 29$, Patrologia Latina 40, col. 246).

24. C. MORRIS, The discovery of the individual, op. cit., p. 31.

25. A. DAMASIO, L'erreur de Descartes, Paris, 1995.

26. É. GILSON, « Moyen Âge et naturalisme ", Archives d'histoire doctrinale et littéraire du Moyen Âge 7, 1932, p. 11-36 (p. 19).

27. PRUDENCE, Psychomachie, v. 840-844, Patrologia latina 60, col. 83.

28. в. BISCHOFF, Anecdota novissima. Texte des vierten bis sechzehnten Jahrhunderts, Stuttgart, 1984 ("Quellen und Untersuchungen zur lateinischen Philologie des Mittelalters », 7), p. 85-90.

29. G. DE PLINVAL, Essai sur le style et la langue de Pélage, suivi du traité inédit De induratione cordis Pharaonis, Fribourg, 1947 (édition : p. 119-203).

30. B. ROSENWEIN, Emotional communities, op. cit., p. 82.

31. B. ROSENWEIN, Emotional communities, op. cit., p. 25.

\section{INDEX}

Mots-clés : Augustin (saint), moi, sujet, individualisme, réflexivité, communauté, anges, tentation, Prudence, De camara Christi, pseudo-Pélage, Ratramne, Florus

\section{AUTEUR}

\section{WARREN PEZÉ}

Université Paris I Panthéon-Sorbonne, LAMOP 